UDC 72.013

\title{
The Notion of Tolerance of the Architectural Form
}

\author{
Negai G.A. \\ Candidate of Architecture, Associate Professor \\ Khmelnytskyi National University
}

\begin{abstract}
The article deals with the new features of the architectural form proposed by the author - tolerance and spaces of tolerance: zones of tolerance and fields of tolerance. They allow you to define strictly the size of the architectural form to harmonize the designed objects of architecture, which can be executed automatically in the process of computer design.
\end{abstract}

Keywords: tolerance, fields of tolerance, tolerance ratio, dimensional structure, informativeness, harmonization.

The concept of tolerance, which clarifies the concept of similarity and the related concept of non-recognizability, was introduced in 1970 by mathematician E. Ziman [1]. The concept of "tolerance ratio" has never been used in the theory of architecture. The term "tolerance space" was first introduced by us in 2000. "Tolerance space" is an architectural form - the set of points of any surface, within which, between any two arbitrarily small zones, distinct visual information of one quality is equal to zero "[2]. This mathematical definition of specific forms requires caution. This is important in view of the future of computer aided design, in the process of which the computer will harmonize the dimensional structure of the architectural form with the preset program. For this computer, you need to specify an algorithm for identifying architectural elements that need to be proportionately matched to achieve a visual perception of harmony.

The topicality of such innovation is determined by the widespread use in the design and construction of architectural objects of various construction and decoration materials with different tonal, color-tone and textural characteristics, which significantly affect the visual complexity of the architectural form, make it indefinitely proportional, distort or alter dimensional structure. This leads to the negative consequences of forming a coherent, information-integrated, harmonious architectural environment. Meanwhile, the researchers of proportions, as a rule, took geometrical parameters of the architectural form as a whole its elements and details, and considered them as elements of the dimensional structure, without 
regard to the patterns of visual perception. But in the process of visual perception our visual apparatus is able to differentiate individual elements of the form, or their visual integration, regardless of geometry or structural and tectonic features. For proportioning the researchers adopted dimensional parameters determined only by the structural parts of the form.

It should be noted that in the study of proportionality at all times they meant only structural or tectonic features of the architectural form, not taking into account its tonal or color characteristics. In addition, whether they wanted it or not, the researchers meant sensual, visually-perceived harmony. For example, B.P Mikhailov, quoting Vitruvius, writes: "The response of the regularity, which goes from individual members to the general form of the whole, is achieved most of all by proportionality, i.e. unity of the ratios of parts, due to which the composition of things acquires the greatest clarity and integrity, causing maximum aesthetic satisfaction "[3, p. 137]. That is, in this fragment B.P Mikhailov, definitely speaks about the sensitive estimation of proportionality, which he interpreted as "the proportionality of all parts with everything", "which Polycletes demanded, and which the ancient Greeks called eurythmy, since each part thus received a harmonious response in proportion to all others and the whole in general'[3, p. 171]. Many researchers have the same opinion. However, what determines the parts, no one has formulated. Below we will try to do this.

First, let us define the concepts of "tolerance zone" and "tolerance field". "Tolerance space" is a generally mathematical concept. Tolerance space can be both a "tolerance zone" within which there are no visually perceived differences in a certain quality, and a "tolerance field" as an association of "tolerance zones" that differs significantly from another architectural field forms in terms of aggregate characteristics and certain quality. In this case, the part of the tolerance field where non-recognizability disappears will be called the boundary of the tolerance field. Physical parameters within the field of tolerance will be called elements of the dimensional structure of the architectural form, its parts.

Consider simple examples of graphical interpretation of these concepts. This will allow us to investigate the similarity and non-recognizability of elements of architectural form. We set the similarity to the tone of a number of rectangles with different intensities. There is a tonal difference between adjacent rectangles (eler is one unit of elementary distinction). The tone modeling of this row of rectangles is accomplished in such a way that each subsequent rectangle becomes darker by one unit of elementary distinction. Thus, a number of rectangles 
has been formed, the neighboring ones are different in tone from each other by the value of the threshold of visual sensation (Fig.1).

Fig. 1. A tonal series of rectangles with a tonal difference of one eler.

Now we will create a tone series in which the first two rectangles will be different in tone by the value of the threshold of visual sensation, and the third rectangle will be toned four or more times darker than the first two. In this tone series, the first two rectangles are combined in a field of tolerance, that is, in such a planar formation that differs substantially from the rectangle in terms of aggregate tone, which is several times darker than the first two (Fig. 2).

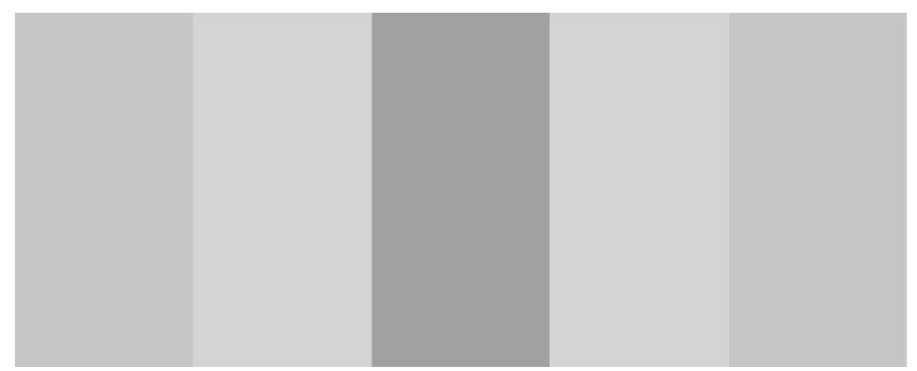

Fig. 2. Tolerance fields.

In this example, you can neglect the tonal difference of two rectangles in one eler compared to a rectangle that is much darker than the first two. It can be claimed that two rectangles with a tonal difference in one eler are in relation to each other's tolerance and are similar in tonality compared to the third rectangle, which is much darker than the first two. In this example, a darker rectangle is antagonistic to the tolerance field of the two lighter rectangles. In practice, a tolerance field can be formed from two or more tolerance zones. Below there are examples of such combinations that determine the visually perceived dimensional structure of an architectural form.

In Fig. 3 and 4 examples of forming tolerance fields with different rectangles are shown. 
In both cases (Fig. 3 and Fig. 4), the formation of a tolerance field occurs on the basis of tonal similarity and non-recognizability, but not uniformity. Equality (equivalence) is a qualitatively different property. It provides for the possibility of replacing one area of the architectural form with another, replacing it, because it repeats all the characteristics of the first: and the tonality, size, and texture, etc. The fact is that the indistinguishable parts of the architectural form are not broken down into separate zones of tolerance, but, on the contrary, are visually combined, forming fields on the principle of similarity or tonal characteristics, or by color, or by some other, for example, similarity in texture, or texture with a consistent tone in tone.

The main task of forming tolerance fields is to determine the parts of the dimensional structure that the designer or the computer working on a given program can include in the process of harmonization, that is, bringing them to a certain system of proportionality with the maximum indicator of the power of information communication [2], taking into account the system of dimension of architectural environment (ensemble) and functional and structural limitations.

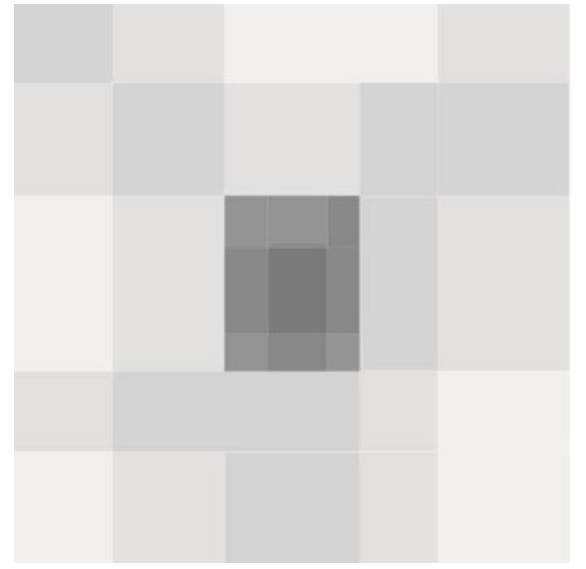

Fig. 3.

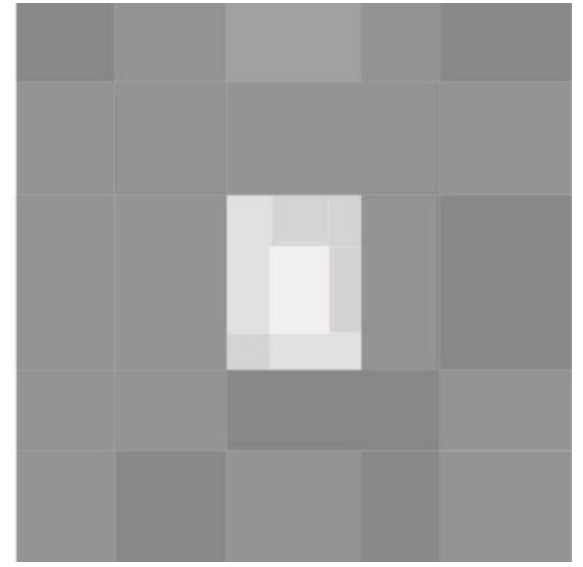

Fig. 4.

Examples of composition formation based on tolerance ratio.

In Fig. 5 depicts a fragment of a facade of a building, which shows that the sheet from the left window to the white pilasters, the pilaster itself and the sheet from the pilasters to the right window are different in tone from each other by the magnitude of the threshold of tonal distinction and are visually combined into a single field of tolerance with respect to a much darker window, both right and left. In turn, windows in different parts have tonal differences 
that can be neglected. Their tolerance zones are combined into a single tolerance field that determines their dimensional structure. In order to analyze the proportionality of the facade, the width of the "wall-pilaster - tolerance field" and the "window" tolerance field width should be taken as elements of the facade structure. Visual information in their ratio will become an informational step of the facade information field, which will be included in the system of determining information modules of the relations of elements of the dimensional structure of the facade and evaluation of the strength of the information (proportional) connection of parts of the architectural form [see. 4]

Another fragment of the facade, shown in Fig. 6, gives us an example of a clearer division into tolerance fields: the window and the shade of the window between the white window frames. Just the size of the windows between the casings and the space between the casings should be taken into account in calculating the proportionality (proportionality) of the parts of this facade. The plinths in this case act as a distributive element. They separate tolerance fields and can be used to adjust their dimensions. And in fig. 7 you can see that the platbands are tonally combined with a tinge of space between them into a single field of tolerance.

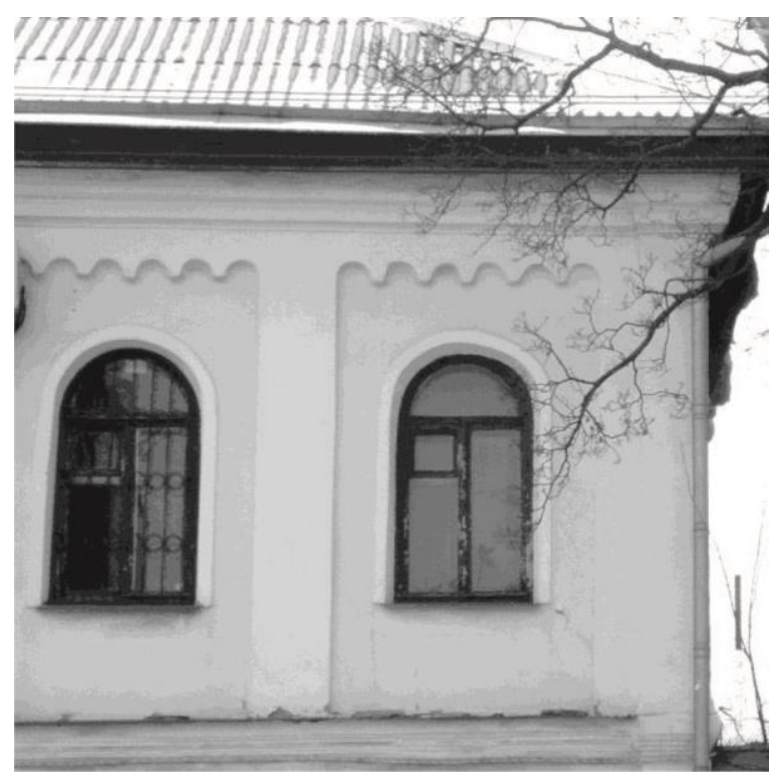

Fig. 5. Depicts a fragment of a facade of a building

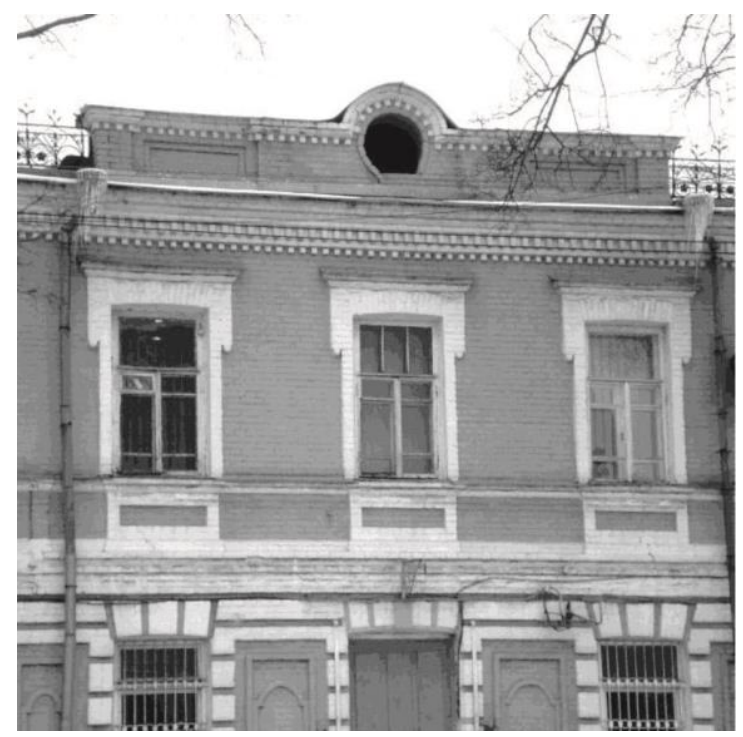

Fig. 6. Fragment of the building facade. Formation of dimensional structure based on tolerance ratio 


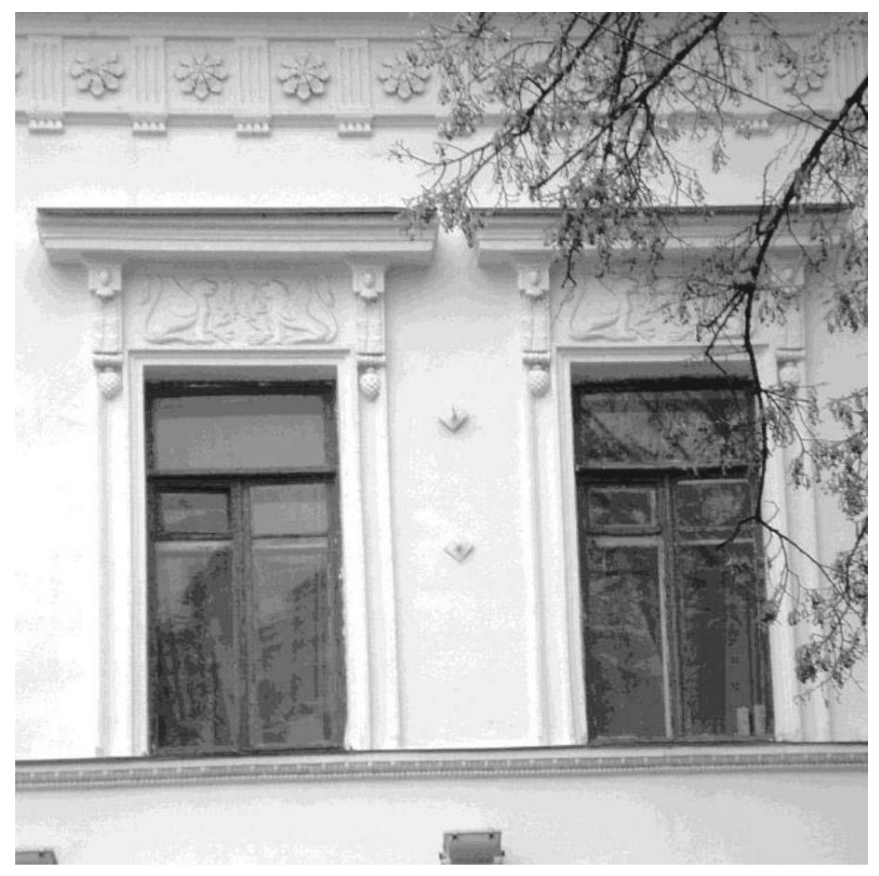

Fig. 7. Fragment of the facade of the building. Façade's tolerance fields.

Conclusion. Based on the concept of tolerance, we can conclude that the parts of the architectural form that should be taken into account when studying the proportionality and harmonization of the architectural form are the fields of tolerance. They determine the visually perceived dimensional structure and form the informative architectural form. The tolerance ratio will make it possible to create an algorithm for computer analysis of the proportionality of the facades of buildings and the harmonization of their dimensional structure. The harmonization of the buildings' facades with regard to the ratio of tolerance will lead to a visual ordering of the architectural form and a significant improvement of their aesthetic quality by increasing the comfort of visual perception.

\section{References}

1. E. Ziman, O. Byuneman. Tolerant spaces and the brain // On the Way to Theoretical Biology. - M.: "World", 1970.

2. G. Negai. Basic provisions of information theory of proportionality // Modern Problems of Architecture and Urban Planning. No. 7. - K.: KNUBA, 2000.

3. B.P. Mikhailov. Vitruvius and Hellas. Fundamentals of the Ancient Theory of Architecture. - M.: 1967.

4. Negai G.A. The information theory of proportionality in architecture // State of modern building science 2006 / IV International Scientific and Practical Internet Conference. Collection of scientific papers. - Poltava, 2006, - p. 225-231. 